EGU21-7440

EGU General Assembly 2021

(c) Author(s) 2021. This work is distributed under

the Creative Commons Attribution 4.0 License.

\title{
Spatial Trends in Mineral Abundances across Tyrrhena Terra on Mars derived from Geomorphological and Mineralogical Mapping
}

\author{
Daniela Tirsch ${ }^{1}$, Joana R. C. Voigt ${ }^{2}$, Christina E. Viviano ${ }^{3}$, Janice L. Bishop ${ }^{4}$, Melissa D. Lane ${ }^{5}$, Livio L. \\ Tornabene, and Damien Loizeau \\ ${ }^{1}$ German Aerospace Center (DLR), Institute of Planetary Research, Berlin, Germany (daniela.tirsch@dlr.de) \\ ${ }^{2}$ Lunar and Planetary Laboratory, University of Arizona, Tucson, AZ \\ ${ }^{3}$ Johns Hopkins University Applied Physics Lab, Laurel, MD \\ ${ }^{4}$ SETI Institute, Mountain View, CA; 5Fibernetics LLC, Lititz, PA \\ ${ }^{5}$ Fibernetics LLC, Lititz, PA
}

Tyrrhena Terra hosts an intriguing variety of aqueously altered materials accompanied by unaltered mafic rocks. Our study region extends from the southern rim of the Isidis impact basin, including the Libya Montes region, southward to the Hellas Basin rim (Fig. 1). The NW part is dominated by lava flows from Syrtis Major that grade southwards into the TT highlands, dissected by fluvial channels and overprinted by abundant impact craters. These landforms together with lobate and fan-shaped deposits within impact craters are evidence for a variable history of erosion and deposition. Ancient phyllosilicate-rich materials have been exposed and uplifted from the subsurface, as they often occur in crater ejecta and central crater uplifts.

Our previous studies used CRISM spectral data together with CTX, HiRISE, and HRSC images as well as their derived topography data to create geomorphological maps of the southern Isidis region and Tyrrhena Terra. These datasets were used to map and characterize the types and occurrences of phyllosilicates, chlorite, opal, zeolites, carbonates, olivines, and pyroxenes and to assess the relationships between selected aqueous outcrops and surface features.

In this work, we build on these results by seeking correlations between aqueous mineral detections with our geomorphological map to assess 1) whether or not there are relationships between specific units and mineral occurrences, and 2 ) if there are trends across the study region in terms of mineral occurrence and abundance.

The mineralogical map originates from a study that spans not only the inter-Isidis-Hellas region, but also extends northwards to Nili Fosse and westwards to Terra Sabea. The focus of that study was on the metamorphic- and hydrothermally-related alteration history using CRISM targeted and mapping data, including hundreds of calibrated MTRDR images. These mineral detections were available to us as a mapped shape file, enabling us to assess the minerals in context with the geomorphological map. We utilized ESRI's ArcGIS system and conducted multiple statistical queries in terms of mineral occurrence/type versus map unit in order to reveal possible trends within and across the study region. 
Fe/Mg-phyllosilicates are the dominant aqueous mineral type within the study region and are more abundant in the central region compared to the proximity of either the Isidis or Hellas impact basin. Chlorites increase in abundance with distance from both impact basins, which could be an indication of hydrothermal processes from geothermal flux. The large Hellas impact event appears to have produced more varied temperatures and water chemistries, resulting in increased mineral variability near its rim. 